Reprod. Nutr. Dévelop. 1980, 20 (4 A), 991-1010.

\title{
L'organe pinéal du Brochet (Esox lucius, L.) \\ $V$. Etude radioautographique de l'incorporation in vivo et in vitro de précurseurs indoliques
}

\author{
par J. FALCON *, Marie-Thérèse JUILLARD, J. P. COLLIN \\ Laboratoire de Zoologie ef Biologie Cellulaire \\ ef Laboratoire Associé ou CNRS no 290 \\ 40, avenue du Recleur Pineau \\ 86022 Poitiers Cedex, France.
}

Summary. The pineal organ of the pike (Esox lucius, L.). V. Radioautographic study of in vivo and in vitro incorporation of indoleaminergic precursors.

The primary aim of the present investigation is to characterize the sites of indole metabolism in the pike pineal organ using a radioautographic approach. The experiments were carried out during the daytime in autumn and winter (short-days).

1) The pike were injected with tritiated tryptophan $\left({ }^{3} \mathrm{H}-\mathrm{TP}\right)$ or tritiated 5-hydroxytryptophan $\left({ }^{3} \mathrm{H}-5-\mathrm{HTP}\right)$ in the air bladder and killed after short survival periods of 10 min to 3 hrs.

2) For in vitro experiments, the pineal organs were incubated for 10 to $20 \mathrm{~min}$ in four different physiological media containing ${ }^{3} \mathrm{H}-5-\mathrm{HTP}$.

Whatever the experimental procedure, a regular labelling pattern was obtained. Depending upon the time-lag between the beginning of incorporation and killing (or fixation), ${ }^{3} \mathrm{H}$-indoleamines were more or less concentrated inside all the receptor line cells (RLC) and interstitial cells (IC). However, the radioautographic reactions were always less in the IC than in the RLC. After ${ }^{3} \mathrm{H}$-TP injection, labelling was particularly reduced and sometimes absent in the IC. The neurons were unlabelled.

3) The pike were given an intraperitoneal injection of ${ }^{3} \mathrm{H}-5-\mathrm{HTP} 20 \mathrm{hrs}$ after nialamide administration. Three hours later, the labelling in the IC as well as in the RLC was strong and diffuse. The reactions decreased after $6 \mathrm{hrs}$ and were very reduced after $9 \mathrm{hrs}$.

4) Using high resolution radioautography, the pineal organs incorporating ${ }^{3} \mathrm{H}-5$-HTP were studied. Although ${ }^{3} \mathrm{H}$-indoleamines were found in the inner segment and cell body, they were mainly distributed in the basal pedicles of the RLC (typical, as well as apparently structurally regressed photoreceptor cells). Silver grains were found (a) in the cytosol or (b) associated with some of the organelles responsible for oxidative deamination (mitochondria, endoplasmic reticular profiles, nuclear envelopes and plasma membranes), the nucleoplasm and bundles of microfilaments, but rarely with the Golgi units. In sections of RLC basal pedicles, silver grains were found in regions containing clear vesicles (35$50 \mathrm{~nm}$ in diameter) or not. However, synapses of the ribbon type did not show constant labelling. The present study did not confirm that an indoleamine (e. g. 5-HT) could be used as a neurotransmitter or that a vesicular compartment would be specialized in indole sequestration.

* Adresse actuelle : Max-Planck-Institut für Physiologische und Klinische Forschung, W. G. Kerckhoff-Instiłut, Parkstrasse 1, 6350 Bad-Nauheim, BRD. 
In IC, the radioautographic reactions were mainly found in cytosol or associated with some of the organelles responsible for oxidative deamination and more rarely in the nucleoplasm.

The significance of most radioautographic reactions has been discussed, taking into account previous histo- and ultracytochemical data on the pike and biochemical data on the indole metabolism in teleosts and other vertebrates. Since the experiments were performed during the daytime, the sites of 5-HTP/5-HT synthesis and storage and those of the oxidative deamination of 5-HT were mainly examined. Methoxy-indole synthesis is also mentioned. The presence of silver grains associated with nucleoplasm, some Golgi units and bundles of microfilaments remained unexplained. It was concluded that RLC as well as IC were implied in the storage and degradation of 5-HT. Only the RLC might be capable of synthesizing 5-HT and methoxyindole(s) (e. g. melatonin). According to this supposition, 5-HT of IC would originate from RLC.

The concept of photoneuroendocrine cells is also examined.

\section{Introduction.}

Ralph (1978) a exposé le rôle joué par l'épiphyse (ou organe pinéal) dans la régulation neuroendocrinienne de la fonction de reproduction des Vertébrés nonmammaliens, dont les Poissons osseux qui nous intéressent ici (voir aussi De Vlaming ef Vodicnik, 1978 ; Vodicnik et De Vlaming, 1978).

La mélatonine, qui participe à ce type de régulation, a été découverte voici plus de vingt ans chez les Amphibiens (Lerner et al., 1958). Elle fut ensuite identifiée dans l'organe pinéal de toutes les classes de Vertébrés, dont un Téléostéen, le Saumon du Pacifique (Fenwick, 1970).

En 1965, Quay, puis Smith ef Weber (1973) mettaient en évidence l'hydroxyindole0-méthyltransférase (HIOMT), enzyme qui catalyse la formation de la mélatonine (et d'autres indolamines: Balemans, 1979), à partir de la $\mathrm{N}$-acétylsérotonine, aussi bien dans la rétine des yeux pairs que dans celle de l'organe pinéal de la Truite. Récemment, Gern et al. $(1978 a, b)$ et Gern et Ralph (1979) onł montré que la mélatonine est, d'une part, synthétisée dans la rétine des yeux pairs de la Truite et, d'autre part, libérée dans l'appareil vasculaire (Gern et Ralph : en préparation). Dans l'hypothèse où la mélatonine mérite réellement le qualificatif d'hormone, Gern et Ralph (1979) suggèrent «the possibility that the trout retina is an endocrine organ ».

Les rétines oculaires et pinéaliennes possèdent des transducteurs hautement spécialisés (ou cellules photoréceptrices) impliqués dans certaines des relations qui s'établissent entre l'environnement et l'organisme. Ils élaborent des messages photosensoriels qui ont été étudiés par les méthodes électrophysiologiques (revues de Dodt, 1973 ; Hamasaki et Eder, 1977 ; pour l'organe pinéal). Le(s) type(s) cellulaire(s) qui élabore(nt) les messages neurohormonaux de type indolique (comme la mélatonine) n'est (ne sont) pas encore découvert(s).

Ełant donné que l'organe pinéal :

1. se caractérise par une pluralité des photorécepteurs, en particulier chez le Brochet (Falcon, 1979b) ; 
2. élabore des indolamines [sérotonine (5-HT) : ref. in Falcon, 1979a ef Falcon et al., 1980 ; mélatonine (MEL) : Fenwick, 1970 ; voir aussi Smith ef Weber, 1974, 1976 pour ['HIOMT] ;

3. possède, comparativement à l'œil, une rétine simplifiée, nous choisissons ce dérivé diencéphalique dorsal, comme modèle d'étude du métabolisme indolique à l'échelle cellulaire.

La découverte du (ou des) type(s) cellulaire(s) impliqué(s) dans ce métabolisme doit contribuer, au niveau d'organes photosensoriels, à mieux saisir le déterminisme des fluctuations circadiennes et circannuelles de principes actifs indoliques comme la mélatonine, ef ceci, dans le cadre de la régulation directe ou indirecte d'effecteurs variés, comprenant l'appareil reproducteur.

La méthode radioautographique, utilisée en complément d'autres techniques (Falcon et al., 1980), pourrait contribuer à répondre au problème ici posé. Nous rapportons, dans le présent article, les résultats préliminaires obtenus pendant la phase diurne, au cours des périodes automnale ef hivernale (jours courts).

\section{Matériel et techniques.}

\section{Matériel biologique.}

29 Brochets (Esox lucius, L.) mâles et femelles, de poids et âge différents, ont été sacrifiés en octobre 1975, février et octobre 1977 (jours courts). Les conditions d'élevage (photopériode nałurelle) furent précisées antérieurement (Falcon, 1979a). A chacune des séries expérimentales suivantes (voir tabl. 1 et 2 ), correspond un lot homogène en poids d'animaux des deux sexes.

\section{Traitements des animaux ef des organes.}

a) Précurseurs radioactifs et drogue. - Nous avons utilisé le tryptophane- ${ }^{3}$ H(TP${ }^{3} \mathrm{H}$; marquage général) et le 5 -hydroxytryptophane- ${ }^{3} \mathrm{H}\left(5-\mathrm{HTP}_{-}{ }^{3} \mathrm{H}\right.$; marquage général) fournis par le "Radiochemical Center » d'Amersham (Grande-Bretagne). Les différentes activités spécifiques de ces précurseurs seront précisées ultérieurement.

L'inhibition des activités monoamine oxydasiques est obtenue par injection de nialamide (Niamide, Laboratoire Pfizer-Clin, France).

b) Séries 1-3, 4-9, $10-15$ : incorporation in vivo de 5-HTP- ${ }^{3} H_{\text {. }}-$ Le tableau 1 résume les conditions expérimentales choisies. Après le sacrifice, les épiphyses sont fixées, déshydratées et incluses pour la microscopie électronique selon un protocole exposé antérieurement (Falcon, 1979a). Les coupes semifines $(1 \mu)$ et ultrafines sont obtenues sur un ultramicrotome Reichert, OMU 2.

c) Série 16-17 : incorporation in vivo de $\mathrm{TP}^{3} \mathrm{H}$ (activité spécifique : $5 \mathrm{Ci} / \mathrm{mMole}$ ). Deux animaux (non traités au nialamide) ont reçu le 4/X/1977 à 16 h 40, une injection de $30 \mu \mathrm{Ci} / \mathrm{g}$, dans la vessie gazeuse. Ils ont été sacrifiés 1 et $2 \mathrm{~h}$ après. Les épiphyses sont ensuite traitées pour la microscopie électronique (voir ci-dessus). 


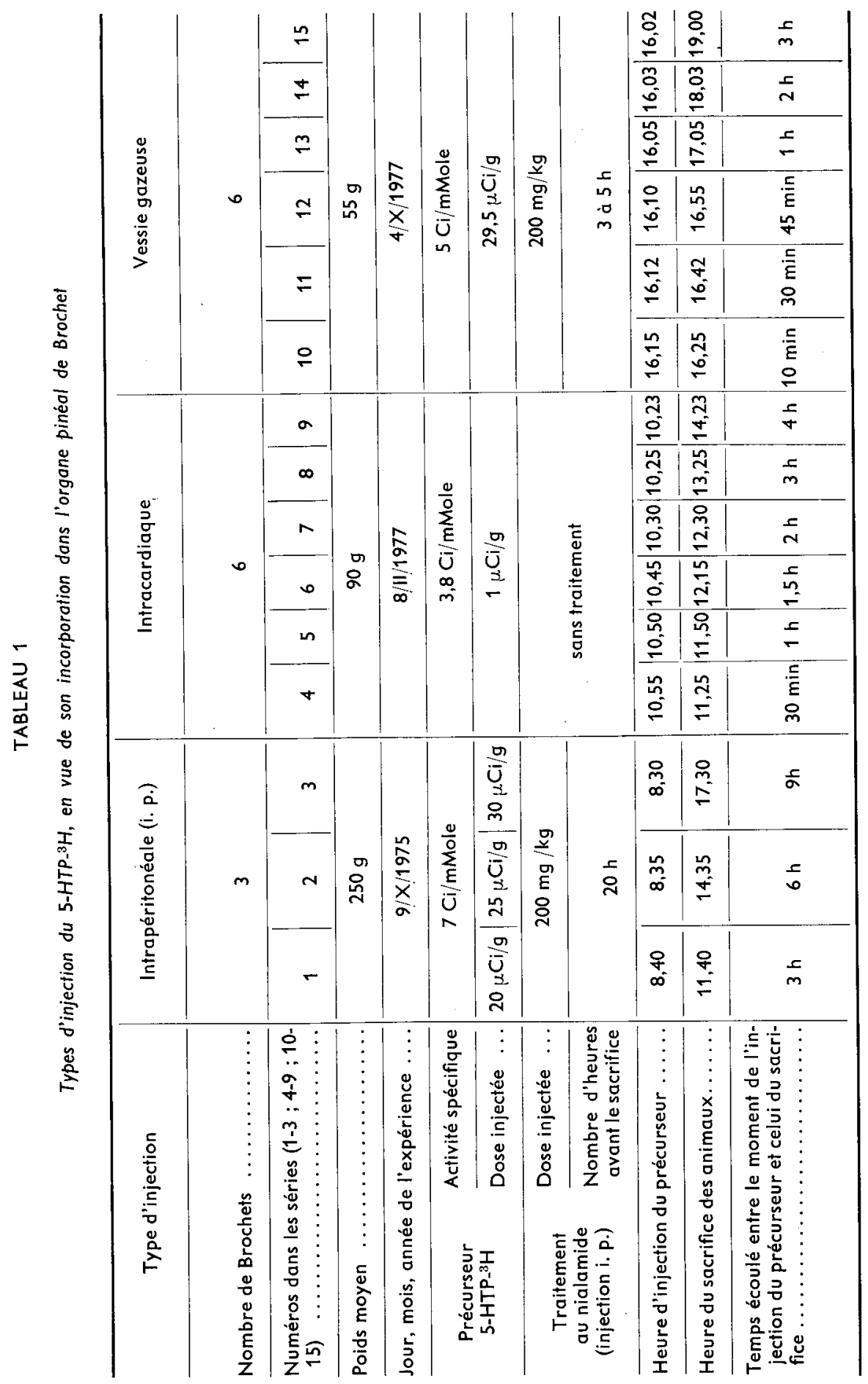


d) Série 18-29: incorporation in vitro de 5-HTP-3 H. - Le tableau 2 résume les techniques utilisées. L'incubation a lieu dans une solution physiologique pour Poissons d'eau douce, contenant le précurseur tritié. Quatre milieux type Ringer (Burnstock, «goldfish», Hanks et Krebs) ont été employés.

TABLEAU 2

Conditions d'incorporation in vitro du $5-H_{T P-}{ }^{3} \mathrm{H}$ dans l'organe pinéal de Brochet

\begin{tabular}{|c|c|c|c|c|c|c|c|c|c|c|c|c|c|}
\hline \multicolumn{2}{|c|}{ Milieux physiologiques } & \multicolumn{3}{|c|}{ Hanks Ringer } & \multicolumn{3}{|c|}{ Burnstock Ringer } & \multicolumn{3}{|c|}{ Krebs Ringer } & \multicolumn{3}{|c|}{$\begin{array}{l}\text { "Goldfish" } \\
\text { Ringer }\end{array}$} \\
\hline \multicolumn{2}{|c|}{ Nombre de Brochets } & \multicolumn{3}{|c|}{3} & \multicolumn{3}{|c|}{3} & \multicolumn{3}{|c|}{3} & \multicolumn{3}{|c|}{3} \\
\hline \multicolumn{2}{|c|}{ Numéros dans la série $18-29$} & 18 & 19 & 20 & 21 & 22 & 23 & 24 & 25 & 26 & 27 & 28 & 29 \\
\hline \multicolumn{2}{|c|}{ Poids moyen ............ } & \multicolumn{3}{|c|}{$175 \mathrm{~g}$} & \multicolumn{3}{|c|}{$145 \mathrm{~g}$} & \multicolumn{3}{|c|}{$165 \mathrm{~g}$} & \multicolumn{3}{|c|}{$175 \mathrm{~g}$} \\
\hline \multicolumn{2}{|c|}{$\begin{array}{l}\text { Jour, mois, année de l'expé- } \\
\text { rience } \ldots \ldots \ldots \ldots \ldots \ldots\end{array}$} & \multicolumn{3}{|c|}{$4 / X / 1977$} & \multicolumn{3}{|c|}{$4 / X / 1977$} & \multicolumn{3}{|c|}{$4 / X / 1977$} & \multicolumn{3}{|c|}{$4 / X / 1977$} \\
\hline \multicolumn{2}{|c|}{ Heure du sacrifice ......... } & 9,38 & 9,43 & 12,55 & 10,10 & 10,20 & 11,43 & 10,52 & 10,57 & 11,55 & 8,50 & 8,55 & 12,30 \\
\hline \multirow{2}{*}{$\begin{array}{l}\text { Précurseur } \\
5-\mathrm{HTP}^{3} \mathrm{H}\end{array}$} & $\begin{array}{l}\text { Activité spé- } \\
\text { cifique } \quad . .\end{array}$ & \multicolumn{3}{|c|}{$5 \mathrm{Ci} / \mathrm{mMole}$} & \multicolumn{3}{|c|}{$5 \mathrm{Ci} / \mathrm{mMole}$} & \multicolumn{3}{|c|}{$5 \mathrm{Ci} / \mathrm{mMole}$} & \multicolumn{3}{|c|}{$5 \mathrm{Ci} / \mathrm{mMole}$} \\
\hline & $\begin{array}{l}\text { Dose } \\
\mathrm{mCi} / 5 \mathrm{ml}\end{array}$ & 0,1 & 0.1 & 0,5 & 0,1 & 0.1 & 0,5 & 0,1 & 0,1 & 0,5 & 0,1 & 0,1 & 0,5 \\
\hline \multicolumn{2}{|c|}{$\begin{array}{c}\text { Durée de l'incubation (en } \\
\min ) \ldots \ldots \ldots \ldots \ldots \ldots \ldots\end{array}$} & 10 & 20 & 20 & 10 & 20 & 20 & 10 & 20 & 20 & 10 & 20 & 20 \\
\hline
\end{tabular}

Dès son prélèvement, l'organe pinéal est placé, pendant $10 \mathrm{~min}$, dans l'un des quatre milieux, à $+4^{\circ} \mathrm{C}$. L'incubation a lieu ensuite à $+^{-} 16^{\circ} \mathrm{C}$ dans une solution identique à laquelle on a ajouté le précurseur tritié, et en présence d'un courant de carbogène. Pour les fixations, l'inclusion ef la confection des coupes, le lecteur se reportera au paragraphe $b$ de cette rubrique.

\section{Radioautographie.}

La technique radioaulographique a été couramment utilisée pour l'étude des neurones sérotonergiques du système nerveux central (réf. in Bequdet et Descarries, 1978), y compris ceux de plusieurs organes circumventriculaires (éminence médiane: Calas et al., 1974 ; organe sous-commissural : Bouchaud et Arluison, 1977 ; organe vasculaire de la lame terminale : Bosler, 1978). Un autre organe circumventriculaire, l'épiphyse, a également fait l'objeł d'études radioautographiques, plus particulièrement chez les Amniotes (réf. in Collin, 1979). Le métabolisme des indolamines dans les cellules très particulières de l'épiphyse est plus complexe que dans les neurones sérotonergiques. 
a) En microscopique photonique (selon Larra et Droz, 1970). - Les coupes semifines sont recueillies sur lames de verre et recouvertes de l'émulsion nucléaire llford $K 5$, diluée de moitié. Après séchage, les lames sont placées dans des boîtes de ClayAdams, en présence d'un agent déshydratant $\left(\mathrm{P}_{2} \mathrm{O}_{5}\right)$ pendant 10,20 ou 30 jours. Les radioautographies sont alors révélées dans le $D 19$ b Kodak, rincées et fixées par le thiosulfate de sodium à 30 p. 100 . Elles sont observées après coloration au bleu de toluidine et montage dans le Permount.

b) En microscopie électronique (selon Larra et Droz, 1970). - Les coupes ultrafines sont recueillies sur lames de verre, préalablement recouvertes d'une couche de celloïdine (solution à 2 p. 100 dans l'acétate d'isoamyle). Elles sont ensuite doublement contrastées à l'acétate d'uranyle, puis au citrate de plomb; après séchage, elles sont recouvertes d'une mince couche de carbone. Les lames sont ensuite plongées dans l'émulsion llford L4 diluée au $1 / 5^{\mathrm{e}}$, à $+40^{\circ} \mathrm{C}$. Après une exposition moyenne de 45 jours, les radioautographies sont révélées par le D 19 b Kodak, rincées et fixées par le thiosulfate de sodium à 30 p. 100.

\section{Résultats.}

1. Mise en évidence des indolamines exogènes par radioautographie en microscopie photonique.

a) Série 1-3. - Le 5-HTP-3 H est injecté par voie intrapéritonéale à des animaux traités $20 \mathrm{~h}$ avant au nialamide.

Les grains d'argent sont toujours répartis de façon homogène, tout particulièrement dans le cytoplasme des cellules de type photorécepteur (CTP) et celui des cellules interstitielles de type épendymaire (CIE) des trois régions de l'organe pinéal (Falcon, 1979a, b). Chez le Brochet sacrifié $3 \mathrm{~h}$ après administration du précurseur $(20 \mu \mathrm{Ci} / \mathrm{g})$, le marquage est très intense dans les deux catégories cellulaires (CTP et $\mathrm{ClE})$, mais surtout à la partie basale de l'épithélium sensoriel. Après $6 \mathrm{~h}(25 \mu \mathrm{Ci} / \mathrm{g})$, la répartition des grains d'argent n'a pas changé, mais leur nombre a diminué. Au terme des $9 \mathrm{~h}(30 \mu \mathrm{Ci} / \mathrm{g})$, le marquage de l'épithélium est, comparativement à celui observé à 3 et $6 \mathrm{~h}$, très faible; il se caractérise toujours par une répartition diffuse.

b) Série 4-9. - Le 5-HTP- ${ }^{3} \mathrm{H}$ est administré par voie intracardiaque chez des Brochets non prétraités au nialamide.

Aucune réaction radioautographique n'a pu être décelée chez les six animaux éłudiés.

c) Série 10-15. - Chez des animaux, traités 3 à 5 h avant au nialamide, te 5-HTP${ }^{3} \mathrm{H}$ est injecté dans la vessie gazeuse.

On observe un marquage de tout l'épithélium épiphysaire, dont l'intensité varie en fonction du temps écoulé entre le moment de l'injection et celui du sacrifice. Dix minutes après l'injection du précurseur, les grains d'argent sont très rares ; après 30,45 ef $60 \mathrm{~min}$, ils sont observés dans les CIE et surtout dans les CTP (fig. 1) ; après $2 \mathrm{~h}$, l'intensité des réactions augmente, mais la répartition cellulaire des grains d'argent demeure inchangée (fig. 2). Lorsque les animaux sont sacrifiés $3 \mathrm{~h}$ après l'administration du précurseur, des réactions radioautographiques très intenses affectent les 2 types cellulaires, mais principalement les CTP. 
Un marquage diffus après incorporation in vivo ou in vitro de $5-\mathrm{HTP}_{-}{ }^{3} \mathrm{H}$, ou de TP-3H (voir ci-dessous) peut avoir lieu dans le sac dorsal (fig. 1).

d) Série 16-17. - Le TP-3 ${ }^{3}$ est injecté dans la vessie gazeuse de Brochets non prétraités au nialamide.

Le marquage concerne tout l'épithélium des régions distale, moyenne et proximale (fig. 3, 4, 5). Quelle que soit l'heure du sacrifice, on constate une accumulation de grains d'argent dans les CTP. Ceux-ci sont beaucoup moins nombreux (fig. 3, 4), parfois rares, voire même absents dans les CIE. Le marquage dans les CTP est particulièrement intense lorsque l'animal est sacrifié $2 \mathrm{~h}$ après l'injection du précurseur (fig. 5).

Il semble que, dans la partie la plus rostrale de la région distale ici explorée, l'incorporation du précurseur ait été légèrement moins importante que dans la région moyenne (comparer fig. 3 et 4 ).

e) Série 18-29 (incorporation in vitro de $5-H T P_{-}{ }^{3} H$ ). - Une incorporation du précurseur a lieu au niveau de tout l'organe pinéal. Les résultats sont identiques, quel que soit le milieu physiologique utilisé (voir aussi discussion).

Le marquage obtenu est hétérogène car, dans certains cas, les tissus qui circonscrivent et accompagnent l'épiphyse (sac dorsal, méninges) lors du prélèvement, peuvent gêner ou retarder, surtout au cours de brèves incubations, la pénétration du précurseur (fig. 6). Lorsque l'incorporation est importante, les CTP montrent une concentration élevée en grains d'argent, signalant la présence de $5-\mathrm{HTP}-{ }^{3} \mathrm{H}$ ef/ou de certains dérivés (fig. 7). A l'opposé, un marquage plus faible caractérise les CIE.

2. Mise en évidence des indolamines exogènes par radioautographie en microscopie électronique.

Après 45 jours d'exposition, nous avons envisagé l'incorporation du $5-\mathrm{HTP}^{3} \mathrm{H}_{\text {, }}$ in vivo (après $3 \mathrm{~h}: \mathrm{n}^{\circ} 15$ ) ef in vitro (après $20 \mathrm{~min}$, à la concentration de $100 \mu \mathrm{Ci} / 5 \mathrm{ml}$ : $n^{0} 29$ ), dans les régions proximale et moyenne des épiphyses préalablement étudiées en microscopie photonique. Quel que soit le protocole expérimental utilisé, les résultats sont très voisins à l'échelle ultrastructurale : ainsi, nos observations peuvent être consignées dans la même rubrique.

En microscopie électronique, d'une part nous confirmons les résultats acquis en microscopie photonique (à savoir une localisation des grains d'argent, plus ou beaucoup plus importante dans les CTP que dans les CIE) et, d'autre part, nous précisons les sites cellulaires des réactions radioautographiques.

a) Localisation des grains d'argent dans les CTP. Des sections favorables, longifudinales, de CTP permettent d'établir (fig. 8) que les réactions radioautographiques sont présentes dans le segment interne, le corps cellulaire et les pédicules basaux. A l'opposé, la présence de grains d'argent dans le segment externe est exceptionnelle.

- Dans le segment interne : les grains d'argent sont très fréquemment superposés aux mitochondries et aux saccules du réticulum (fig. 8,9 ). Ils sont également présents dans le hyaloplasme et occasionnellement associés aux saccules des dictyosomes (fig. 10).

- Dans le corps cellulaire : les réactions radioautographiques apparaissent au niveau du nucléoplasme et de l'enveloppe nucléaire (fig. 8). Les grains d'argent sont égale- 


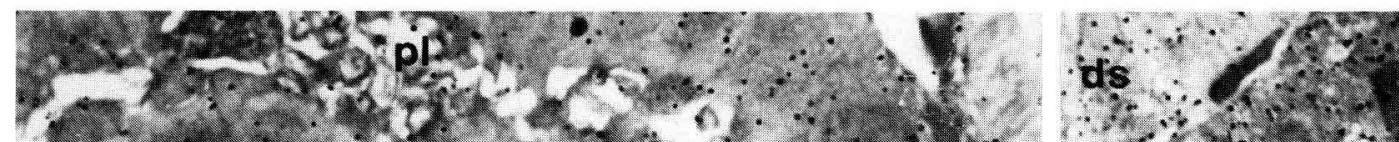

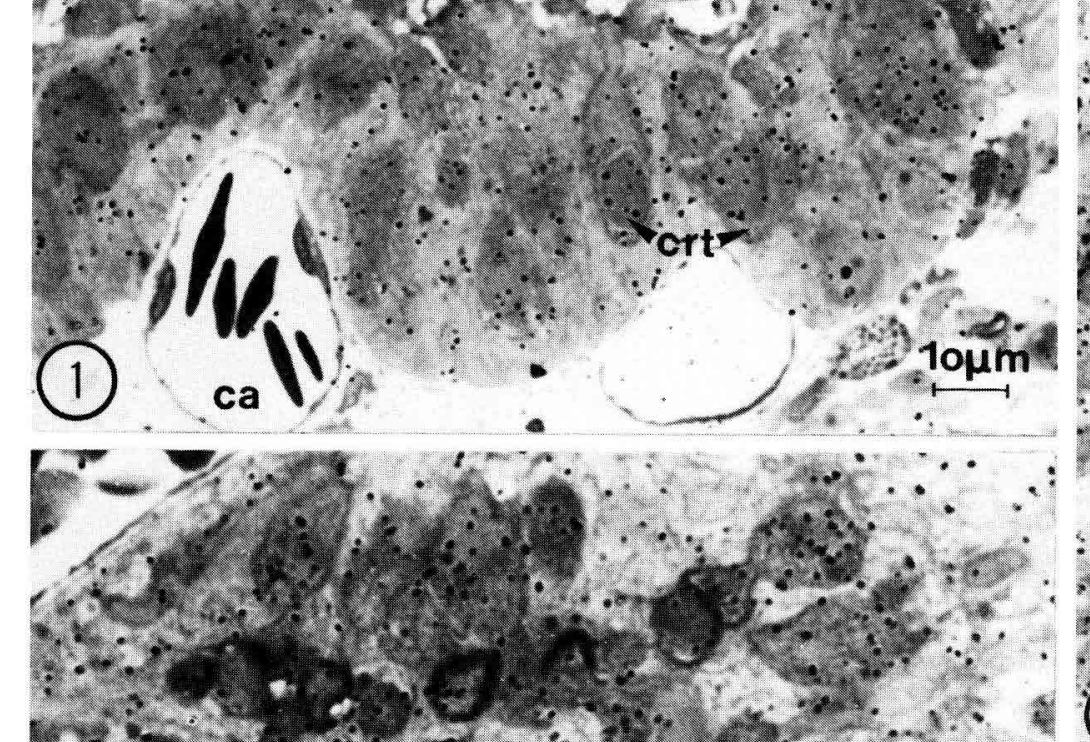

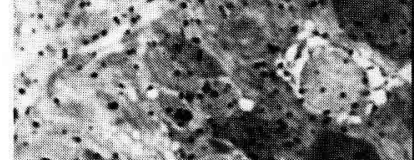
$x+2,1$

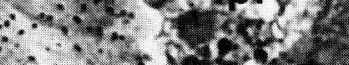

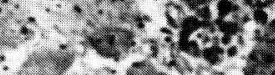
$\because 3 . \div 5,-2$ $\therefore-3 y^{3}$ है

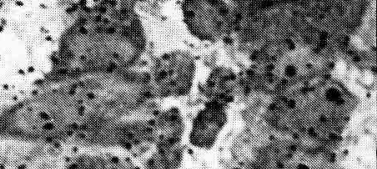

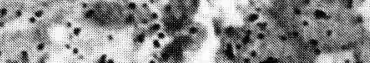

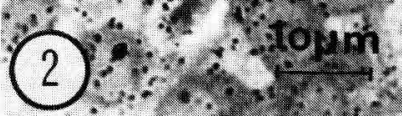

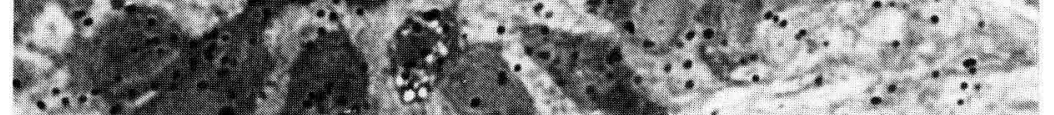

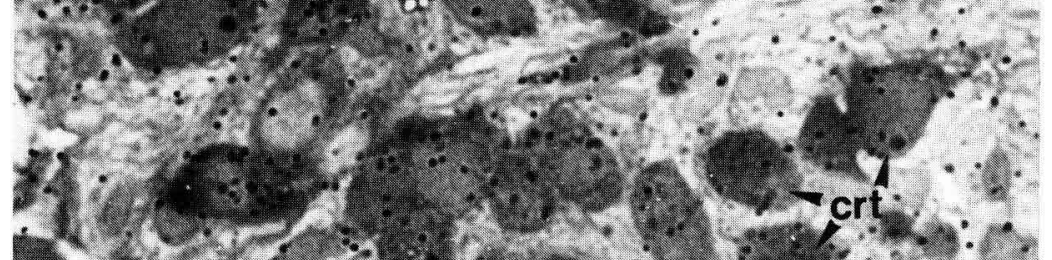
1. - -243

(

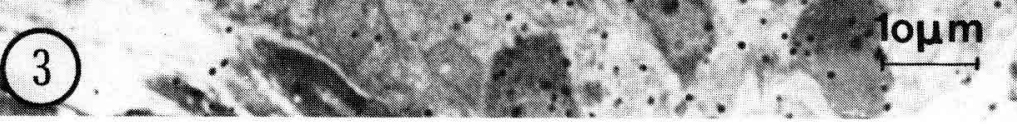
arit? 45

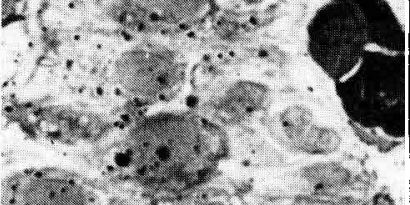

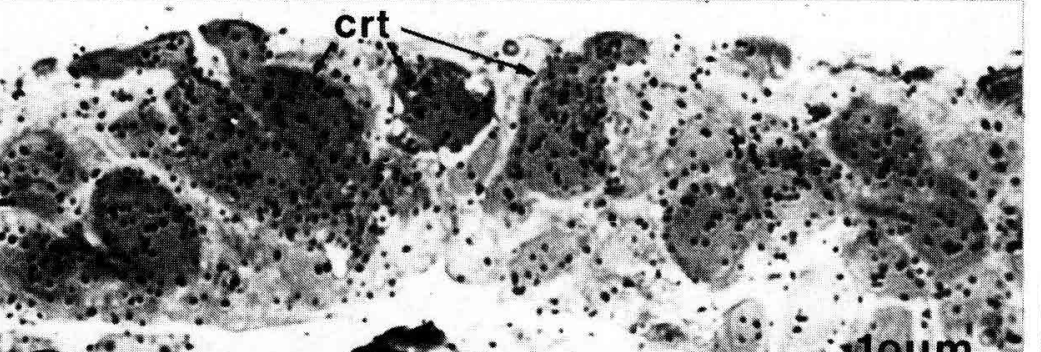

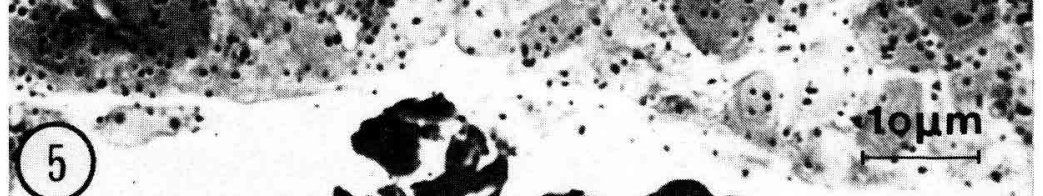
(2. -52 (5) $\quad-2$ (4)

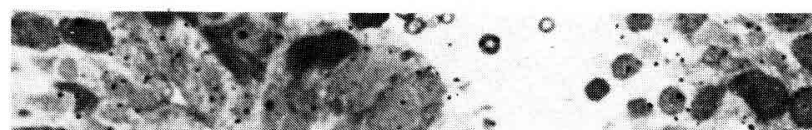
$6-5)(20)$ $6 x^{6}+3 x^{2}$ 
ment libres dans le hyaloplasme périnucléaire (fig. 11) et très souvent localisés dans les faisceaux de micrafilaments (fig. 11). De plus, comme dans le segment interne, ils peuvent être associés aux milochondries et au réticulum.

- Dans les pédicules basaux : malgré l'absence d'étude quantitative, le marquage apparaît régulièrement plus intense dans les pédicules que dans les autres régions des CTP. II concerne les sections de prolongements riches en «vésicules claires》 (de 350 à $500 \AA$ de diamètre) aussi bien que celles qui en sont pauvres, voire même dépourvues (fig. 12,13). Les grains d'argent se superposent ou non (dispersés dans le hyaloplasme) aux vésicules (fig. 12). Comme dans les autres régions des CTP, ils sont associés aux mitochondries, au réticulum et aux faisceaux de microfilaments (fig. 13). D'autres sections riches en vésicules claires ne sont pas marquées (fig. 13).

Dans la région proximale, nous avons également noté une radioactivité dans des pédicules caractérisés par leurs terminaisons à rubans synaptiques typiques (Falcon, 1979b) établissant des contacts fonctionnels avec les dendrites des neurones sensoriels pinéalofuges. Ces rubans et vésicules associées (fig. 14) ne montrent pas de marquage régulier.

Des grains d'argent sont présents également au niveau de la membrane plasmique.

Des CTP, en cours de dégénérescence (Falcon, 1979b) présentent aussi (fig. 15) la propriété de rétention d'indolamines tritiées.

\section{PLANCHE}

Organe pinéal de Brochet : radioautographie en microscopie photonique

FIG. 1 à 2 . - Incorporation in vivo de 5-HTP- ${ }^{3} \mathrm{H}$ dans la région moyenne de l'organe pinéal de Brochef (série 10-15). Les animaux ont été sacrifiés respectivement $1 \mathrm{~h}$ (fig. 1 ) et $2 \mathrm{~h}$ (fig. 2) après l'administration du précurseur. Dans les 2 cas, le marquage est présent dans les cellules de type photorécepteur $(c r t)$, les plus contrastées, et les cellules interstitielles (les plus claires). Noter qu'il est plus important dans la crt. Après $2 \mathrm{~h}$, le marquage est plus intense. (ca : capillaires ; $d s:$ sac dorsal ; pl : lumière épiphysaire). Fig. 1 ( $\times 756) ;$ Fig. 2 ( $\times 958)$.

FIG. 3, 4à 5. - Incorporotion in vivo de $T P_{-}{ }^{3} \mathrm{H}$ (série 16-17). Fig. 3. Région moyenne (animal sacrifié $1 \mathrm{~h}$ après administration du précurseur) ; les grains d'argent sont essentiellement localisés dans les cellules de type photorécepteur $(c r f)(\times 958)$. Fig. 4. Région distale (animal sacrifié $1 \mathrm{~h}$ après administration $d u$ précurseur) ; comme précédemment le marquage, qui semble légèrement moins intense, est essentiellement localisé dans les cellules de type photorécepteur $(c r i)(\times 907)$. Fig. 5. Région moyenne (animal sacrifié 2 h après administration du précurseur) ; le marquage est très intense dans les cellules de type photorécepteur (crt); quelques grains d'argent sont localisés dans les cellules interstitielles $(\times 1210)$.

FIG. 6 à 7. - Incorporation in vitro de 5-HTP- ${ }^{3} \mathrm{H}$ (milieu physiologique : "goldfish » Ringer ; précurseur : $100 \mu \mathrm{Ci} / 5 \mathrm{ml}$; incubation : $20 \mathrm{~min}$ ). Dans le premier cas (fig. 6), la pénétration du précurseur est faible (voir texte) ; le marquage semble plus particulièrement concerner les cellules de type photorécepteur (flèches), ( $\times 756$ ). Dans le deuxième cas (fig. 7), la pénétration du précurseur est importante; les grains d'argent sont plus nombreux dans les cellules de type photorécepteur $(c r t)(\times 756)$ 
b) Localisation des grains d'argent dans les CIE. De façon générale, les réactions radioaulographiques faibles ou beaucoup plus faibles que dans les CTP, peuvent intéresser le hyaloplasme, les mitochondries, l'enveloppe nucléaire, les citernes du réticulum granulaire et plus rarement le nucléoplasme (fig. 16, 17).

c) Autres localisations des grains d'argent. Les réactions radioautographiques n'ont pu être observées dans les neurones sensoriels. Par contre, quelques grains d'argent apparaissent dans l'espace péricapillaire (près de la lamelle basale) dans l'endothélium et la lumière des capillaires.

\section{Discussion}

1. Conditions d'utilisation, intérêf et limites de la technique radioautographique.

Les méthodes d'étude ici choisies et le problème du temps nécessaire à l'incorporation des précurseurs ont été discutés à l'occasion de recherches sur l'épiphyse des Sauropsidés (Collin et Meiniel, 1973a, b ; Collin ef al., 1976). Chez le Brochet, l'administration intrapéritonéale ou dans la vessie gazeuse des précurseurs a permis leur incorporation au niveau de l'organe pinéal. Par contre, les injections intracardiaques ont conduit à des résultats négatifs, probablement à cause de trop faibles doses de 5-HTP-3H administrées (voir aussi Collin et al., 1976, chez les Oiseaux). In vitro, les différents milieux physiologiques de Ringer ont donné satisfaction : la conservation des tissus est toutefois plus correcte avec le «goldfish 》 Ringer (voir illustrations correspondantes : PI. II ef III).

Jusqu'ici, chez les Poissons, seuls Hafeez et Zerihun (1976) ont effectué un travail similaire. Après injection intracardiaque de $5-\mathrm{HTP}_{-}{ }^{3} \mathrm{H}$ et de sérotonine tritiée $(5-\mathrm{HT}$ ${ }^{3} \mathrm{H}$ ), ils ont observé un marquage diffus dans «les cellules de type photorécepteur et

\section{PLANCHE ॥ \\ Organe pinéal de Brochef : radioautographie en microscopie électronique}

FIG. 8. - Incorporation in vitro de 5-HTP- ${ }^{3} H$ (épiphyse moyenne). A ce faible grossissement, le marquage concerne essentiellement les cellules de type photorécepteur (CRT). La répartition des grains d'argent (cercles) est indiquée dans le texte. (el : mitochondries de l'ellipsoïde ; $K$ : cellule interstitielle ; is : segment interne ; $n$ : noyau; ne : enveloppe nucléaire ; PP : pédicule basal) $(\times 6000)$.

FIG. 9. - Incorporation in vitro de $5-H_{T P-}{ }^{3} H$ (épiphyse moyenne). Partie apicale du segemnt interne. A un grossissement élevé, on remarque que les grains d'argent sont associés aux mitochondries de l'ellipsoide (el) et aux canaux du réticulum (ere). (cs : segment connectif) $(\times 9600)$.

FIG. 10. - Incorporation in vivo de 5-HTP- ${ }^{3} \mathrm{H}$ (épiphyse proximale). Région du myoïde. Les grains d'argent sont associés aux mitochondries $(m)$, au réticulum (ere) ; ici un marquage est également enregistré au niveau des saccules d'un dictyosome $(g)(\times 26400)$.

FIG. 11. - Incorporation in vivo de 5-HTP- ${ }^{3} H$ (épiphyse proximale). Corps cellulaire. Des grains d'argent sont associés aux faisceaux de microfilaments $(\mathrm{m} f)$ des cellules claires et sombres de type photorécepteur (CRT) ou semblent libres dans le hyaloplasme (tête de flèche). Un grain est également observé dans l'une des deux cellules interstitielles (IC). (n : noyau) $(\times 19200)$. 


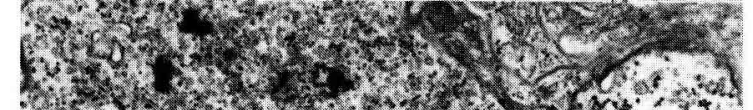
T. 2.8. on 10 7. 1 . 150

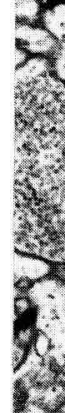
(11) - 201210 1 . $016 T^{5}$ - $1.7 .1 \%$ $2.20,1,15$ (2): 1.7 1 (4) do O 146

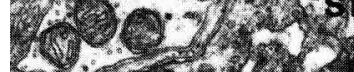

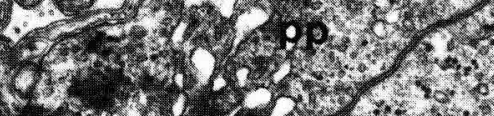
(14) 20.5 D. 4 ic $\left.y^{2}\right)$ 2.7 cis. 1 6. ints. (1) 3 , 1 , o a. 7.25

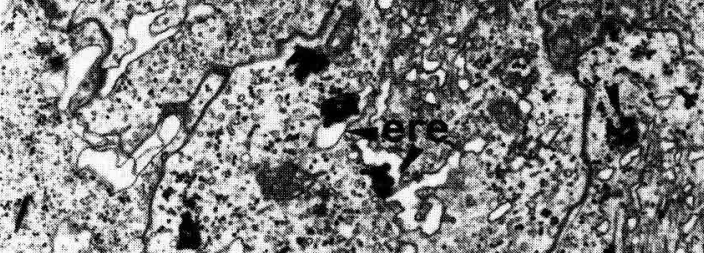
$2 y^{2}$

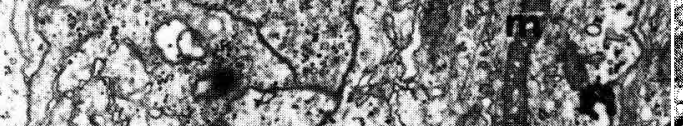

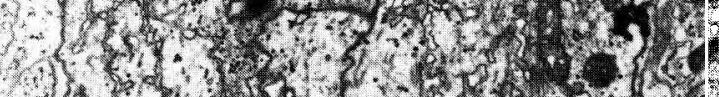
2. . 2 25.

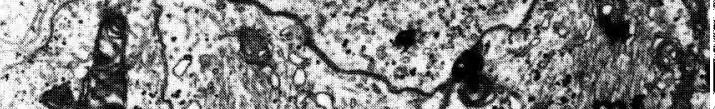

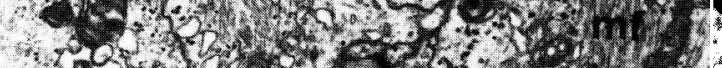
. $1070^{\circ}$ (3)

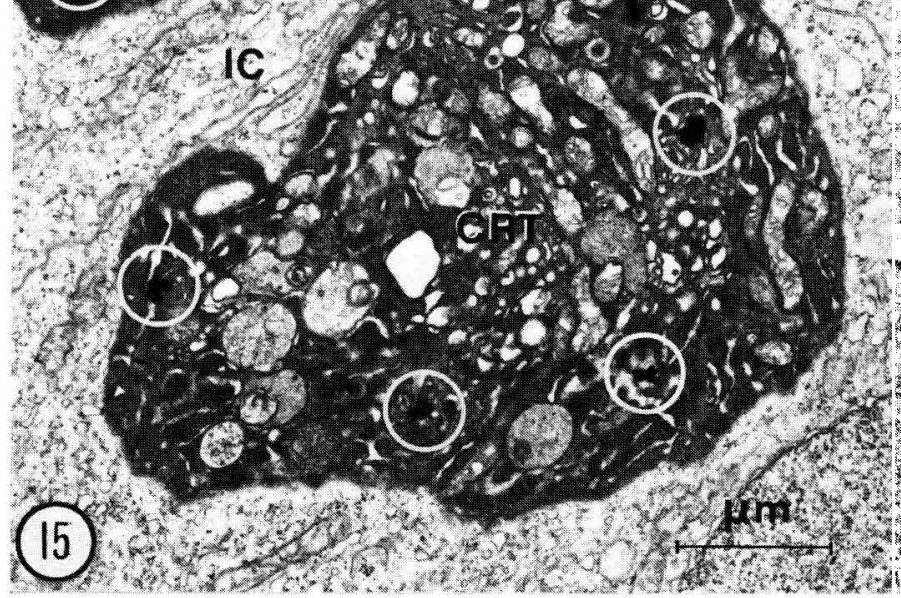

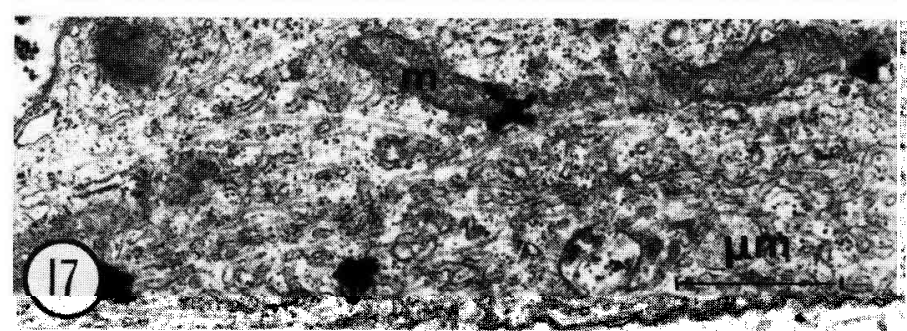


de soutien » de l'organe pinéal de la Truite. Les conditions de fixation et d'enrobage de nos organes sont celles qui sont généralement reconnues pour être les plus favorables à la préservation des sites monoaminergiques (Gershon et Ross, 1966 ; réf. in paragraphe 3 de «Matériel et Techniques »). Chez la Truite, de telles conditions ne semblent pas avoir été respectées.

Dans le présent travail, nous nous sommes limités au choix de protocoles expérimentaux, susceptibles in vivo et in vitro de satisfaire à la détection des types cellulaires, impliqués dans l'«uptake» et le métabolisme du TP- ${ }^{3} \mathrm{H}$ ef du 5-HTP-3 H. Cependant, il apparaît clairement que l'interprétation biochimique des réactions radioautographiques ici obtenues ne pourra être fournie définitivement que lorsque les méthodes radioautographiques et chromatographiques pourront être couplées.

\section{Etude comparative de la localisation des indolamines endogènes et exogènes.}

Dans un récent article de synthèse (Collin, 1979), nous avons pu constater les relations étroiłes existant entre les données d'histofluorescence (méthode de Falck ef Hillarp) et celles de la radioautographie. Une telle concordance se retrouve également chez le Brochet, car les incorporations in vivo (série 10-15) et in vitro (série 18-29) de $5-\mathrm{HTP}_{-}{ }^{3} \mathrm{H}$ sont à l'origine de réactions radioautographiques qui correspondent étroitement aux sites de stockage de 5-HTP/5-HT (Falcon et al., 1980). Quelle que soit leur origine endogène ou exogène, les indolamines se trouvent principalement loca-

\section{PLANCHE III}

Organe pinéal de Brochet : radiooutographie en microscopie électronique

FIG. 12. - Incorporation in vitro de 5-HTP- ${ }^{3} \mathrm{H}$. Epiphyse moyenne : zone des pédicules. Un marquage relativement important est enregistré dans les pédicules riches en vésicules claires (de 350 à $500 \AA$ de diamètre) $(\times 12800)$.

FIG. 13. - Incorporation in vitro de $5-H T P-3 H$. Epiphyse moyenne : zone des pédicules. Ici, des sections de pédicules, riches en vésicules claires ne sont pas marquées. A l'inverse, dans des sections de pédicules pauvres en vésicules, de nombreux grains d'argent sont observés, soit dans le hyaloplasme (têtes de flèches), soit associés aux mitochondries $(\mathrm{m})$ et au réticulum (ere). ( $\mathrm{mf}$ : microfilaments) $(\times 9600)$.

FIG. 14. - Incorporation in vitro de $5-H T P-{ }^{3} H$ : pédicule synaptique dans la région proximale, dépourvu de marquage au niveau de la région des rubans synaptiques (sr) : un grain d'argent est visible dans la zone plus proximale du pédicule synaptique ( $p b$ ) (voir aussi fig. 12 ef 13). (dd : dendrite de neurone sensoriel) $(\times 18000)$.

FIG. 15. - Incorporation in vitro de $5-H T P-{ }^{3} H$ dons une cellule de type photorécepteur (CRT) en voie de dégénérescence (épiphyse moyenne). Ici aucun marquage n'est visible dans les cellules interstitielles (IC) $(\times 16000)$.

FIG. 16. - Incorporation in vivo de $5-H T P{ }^{3} H$ dans une cellule inferstitielle $(I C)$ de la région proximale. Un grain d'argent semble libre dans le hyaloplasne, un autre est associé à une ciferne du réticuIum granulaire (cis). ( $n$ : noyau) $(\times 36000)$.

FIG. 17. - Incorporation in vitro de $5-H T P-{ }^{3} \mathrm{H}$ dans une cellule interstitielle de la région moyenne. Les grains d'argent se localisent dans le hyaloplasme et au niveau des mitochondries $(m)(\times 16800)$. 
lisées dans les différentes catégories de CTP et à un taux moins important dans les CIE. De plus, lorsque la désamination oxydative est largement et plus vraisemblablement complètement inhibée (Falcon ef al., 1980, et série 1-3 du présent travail), les $\mathrm{CIE}$, autant que les CTP, montrent une très forte charge en indolamines (principalement 5-HT). Néanmoins, les différences entre région distale, d'une part, et régions moyenne et proximale, d'autre part (Falcon et al., 1980) sont ici peu apparentes (voir cependant série 16-17). Il est ainsi possible de conclure (voir aussi Falcon et al., 1980) que les indolamines endogènes et exogènes sont métabolisées dans les différents compartiments de l'organe pinéal du Brochet. Lorsque l'étape de la désamination oxydative n'est pas inhibée, il apparaît clairement que les CTP surtout, mais également les CIE, sont impliquées dans le métabolisme indolique. Quelle est alors la part qui revient à chacune des catégories cellulaires dans ce type de métabolisme? Afin d'apporter des éléments de réponse à cette question importante (voir introduction), il est nécessaire, tout d'abord, de prendre en considération la pluralité des voies de transformation de la 5-HT, selon un mécanisme enzymatique (fig. 1 in Balemans, 1979 ; Collin, 1979).

\section{Résumé des données antérieures nécessaires à l'interprétation des résultats.}

Schématiquement, il est établi (surtout chez les Vertébrés supérieurs : réf. in Wurtman et al., 1968 ; Quay, 1974 ; Balemans, 1974, 1979 ; Collin et al., 1976) que la 5-HT, produite à partir du tryptophane, peut être méthylée ef donner la 5-méthoxytryptamine, ou catabolisée en acide 5-hydroxyindole acétique (A-5-HIA), ou anabolisée pour aboutir à la formation de mélatonine (MEL) ou de 5-méthoxytryptophol (5-MTL), selon deux voies métaboliques différentes, ayant en commun l'étape terminale de la méthylation (par l'intermédiaire de l'HIOMT). Chez les Téléostéens (réf. in Falcon, 1979a ; Falcon et al., 1980), les données sur le métabolisme indolique sont réduites ; cependant, la 5-HT, la MEL ef l'HIOMT ont pu être identifiées (voir aussi Introduction).

Les variations nycthémérales des synthèses et activités enzymatiques (fig. 4 , in Balemans, 1979) permettent de justifier celles du taux des différentes indolamines (Falcon ef al., 1980, pour les variations du taux de 5-HTP/5-HT). De plus, selon la période du nycthémère considérée, une voie donnée du métabolisme se manifeste de façon plus ou moins préférentielle. Ainsi, chez les Oiseaux et les Mammifères, l'élaboration de la MEL a lieu surtout de nuit. Chez la Truite (Salmo gairdneri), l'activité HIOMT (Smith et Weber, 1976) est maximale (pour une photopériode 8L-16D ou 12L-12D, c'est-à-dire voisine de celle utilisée dans nos expériences) au cours des quatre premières heures de la scotophase.

Lorsque les réactions radioautographiques affectent au cours du temps - comme chez certains Sauropsidés (réf. in Collin, 1979) - une seule catégorie cellulaire, l'interprétation des résultats est relativement aisée. Au contraire, lorsqu'elles s'observent à la fois dans les CTP ef les CIE, la part qui revient à chacun des types cellulaires dans le métabolisme indolique, est délicate à élucider.

Dans l'état actuel de nos connaissances, notre interprétation prendra en considération une somme de données cytochimiques (déjà acquises chez le Brochet), biochimiques (obtenues chez les Téléostéens ef Amniotes : voir ci-dessus) et cytologiques (notion d'homologie). En effet, compte tenu : 
a) de l'homologie entre photorécepteurs, photorécepteurs rudimentaires et pinéalocytes dans l'organe pinéal des Vertébrés (concept de lignée des cellules réceptrices : Collin, 1969, 1971, 1977, 1979) ;

b) de l'existence d'une lignée des cellules interstitielles de type épendymaire (Collin et Oksche, en préparation) ;

c) de ce que le métabolisme indolique caractérise l'organe pinéal de toutes les classes de Vertébrés,

il est raisonnable de considérer dans chaque lignée cellulaire des analogies au niveau des sites du métabolisme indolique.

\section{Interprétation des résultats en microscopie photonique.}

Les CTP et les CIE (qui contrairement aux CTP offrent une très grande surface de contact avec la lamelle basale: Falcon, 1979a) captent in vivo le TP- ${ }^{3} \mathrm{H}$ (série 16-17). La rétention du précurseur (et/ou de ses dérivés tritiés) est touł particulièrement importante dans les CTP, parfois absente dans les CIE. Théoriquement, le TP- ${ }^{3} \mathrm{H}$ pourrait être utilisé aussi bien pour les synthèses de protéines variées que pour celles d'indolamines. Pour cette raison, la nature des molécules marquées (protéiques ? et indoliques), largement dispersées dans les CTP, ne peut être précisée en l'absence d'étude biochimique parallèle, qualitative et quantitative, des indoles. Actuellement, la période du nycthémère où s'accomplit préférentiellement la première étape de la transformation du TP en 5-HTP est inconnue.

L'avantage que présente le $5-\mathrm{HTP}_{-}{ }^{3} \mathrm{H}$ par rapport à son précurseur $\left(\mathrm{TP}^{3} \mathrm{H}\right)$ est qu'en majeure partie (ou en totalité ?) il est engagé dans le métabolisme indolique (Collin et Meiniel, 1973a, b). Les réactions radioautographiques (séries 10-15, 18-29) sont régulièrement présentes dans les CTP et les CIE, toujours plus intenses dans la première catégorie cellulaire que dans la seconde. L'incorporation in vivo (série 10-15) et in vitro (série 18-29) de 5-HTP- ${ }^{3} \mathrm{H}$ a eu lieu en période diurne, c'est-à-dire dans les conditions où le taux de $5-\mathrm{HT}$ endogène est maximum chez de nombreuses espèces, $y$ compris chez le Brochet (Falcon et al., 1980). De plus, compte tenu de nos conditions expérimentales, des données de Wilhoft et Quay (1965) et de celles de la série 1-3, il est raisonnable de concevoir que la décarboxylation (Falcon et al., 1980) du 5-HTP-3 H conduise à la formation de $5-\mathrm{HT}_{-}{ }^{3} \mathrm{H}$, dans l'organe pinéal de Brochet. Les résultats obtenus après inhibition expérimentale de la désamination oxydative (série 1-3), suggèrent l'existence d'une surcharge anormale de $5-\mathrm{HT}^{3} \mathrm{H}$ (après $3 \mathrm{~h}$ ) dans les CTP et les CIE et, en conséquence, une dégradation importante de la $5-\mathrm{HT}$, dans les conditions normales.

\section{Interprétation des résultats en microscopie électronique.}

II est possible d'apporter une interprétation, qui reste néanmoins préliminaire, aux réactions radioautographiques, observées en microscopie électronique, après incorporation du 5 - HTP_ $3 \mathrm{H}$, in vitro et in vivo (séries $10-15 ; 18-29)$. Considérant la localisation des sites de l'activité monoamine oxydasique (MAO) (Falcon et al., 1980) et des conclusions récemment proposées par Juillard et Collin (1979), les grains d'argent des CTP et CIE se rapportant aux mitochondries, enveloppes nucléaires, citernes du réticulum et membranes plasmiques suggèrent qu'ils représentent des 
indolamines tritiées de type 5-HT et/ou des dérivés de sa désamination oxydative. En effet, dans les expériences in vitro (série 18-29), le nialamide (inhibiteur des MAO) n'a pas été utilisé et in vivo (série 10-15), son temps d'action est trop bref pour permettre d'envisager une inhibition complète (réf. in Collin et Meiniel, 1973b; voir aussi Falcon et al., 1980). Le marquage des membranes plasmiques pourrait correspondre également au phénomène de transfert de molécules indoliques entre cellules de même catégorie ou de catégories différentes (voir ci-dessous : mécanisme de diffusion de la 5-HT). Quant aux grains d'argent des CTP, localisés dans le cytosol, ils pourraient correspondre à des molécules de 5-HTP, mais plus vraisemblablement à des molécules de 5-HT (voir ci-dessus) d'autant que l'activité 5-hydroxytryptophane-décarboxylasique ne paraît pas siéger au niveau d'organites particuliers (Klein, Illnerova, communication personnelle), mais plutôt dans le cytosol.

Dans le cadre de nos recherches, effectuées jusqu'ici uniquement au cours de la photophase, il est délicat (voir conclusion) de prendre en considération au niveau des CTP, les sites hyaloplasmiques d'acétylation (N-acetyl-transférase : NAT) de la 5-HT et de méthylation (HIOMT) de la $\mathrm{N}$-acetyl-sérotonine, en vue de la formation de mélatonine. Certes, la production de mélatonine chez la Truite peut s'effectuer de jour (mais surtout de nuit : fig. 1 , in Smith et Weber, 1976). De plus, nous ignorons chez les Téléostéens, le temps nécessaire aux molécules de TP- ${ }^{3} \mathrm{H}$ ou de $5-\mathrm{HTP}^{3} \mathrm{H}$ pour être transformées, dès leur «uptake », en MEL- ${ }^{3} \mathrm{H}$.

De l'expérience in vitro (série 1-3), il ressort que les résultats sont qualitativement et quantitativement différents de ceux obtenus par Collin et Meiniel $(1973 \mathrm{~b}) \mathrm{chez}$ Lacerta, également traité au nialamide ; ils suggèrent, en considérant le marquage obtenu 9 heures après l'injection du précurseur, que le «turn over » des indolamines est relativement rapide chez le Brochet. Néanmoins, dans les conditions expérimentales présentes, il esł délicat d'apprécier la part de $5-\mathrm{HT}_{-}{ }^{3} \mathrm{H}$ engagée dans les voies métaboliques conduisant à la formation de méthoxy-indoles, et/ou libérée.

La signification du marquage du nucléoplasme (voir aussi Collin, 1979), de même que celui de dictyosomes ef surtout de faisceaux de microfilaments ne peut être fournie dans l'état actuel de nos recherches. Il est intéressant de noter également que les réactions radioautographiques dans les CTP sont particulièrement importantes dans les pédicules, propriété déjà retenue au niveau des cellules de la lignée réceptrice de l'organe pinéal des Amniotes (réf. in Collin, 1979). Cependant, compte fenu de la technique utilisée et des résultats ici obtenus, il n'est pas possible de suggérer que le matériel vésiculaire possède, en particulier, la propriété de rétention des indolamines et que les synapses de type ruban (Falcon, 1979b) utilisent comme neurotransmetteur une indolamine (par exemple la 5-HT).

Dans les CIE, la signification de la radioactivité du cytosol peut être justifiée de deux manières:

a) la première rejoint celle ci-dessus exposée pour les CTP ;

b) la seconde prend en comple les données de phylogenèse structurale ef fonctionnelle des CIE (Collin, 1979 ; Collin et Oksche, en préparation) : les indolamines- ${ }^{3} \mathrm{H}$ des CIE correspondraient à des molécules de $5-\mathrm{HTP}^{3} \mathrm{H}$ récemment incorporées et non métabolisées (voir le présent protocole expérimental ef la discussion de Collin ef Meiniel, $1973 a, b)$ et/ou de $5-\mathrm{HT}_{-}{ }^{3} \mathrm{H}$ en provenance des CTP et destinées à subir la désamination oxydative. 
Cette seconde hypothèse nous paraît plus vraisemblable. En effet, chez le Brochet, à l'inverse de Sauropsidés (Collin, 1979), il n'y a pas - ou seulement dans une très faible proportion - d'u uptake » de la 5 -HT par les pièges à indoles (du type vésiculaire à cœur dense ou autre). En conséquence, l'absence de ce type de stockage pourrait être favorable (voir aussi Collin, 1979) à une diffusion de la 5-HT, des CTP vers les $\mathrm{CIE}$, et éventuellement à sa libération extracellulaire. La surcharge expérimentale des $\mathrm{CIE}$ en indolamines (principalement 5-HT) d'origine endogène (Falcon et al., 1980) ou exogène (série 1-3) chez des animaux où l'activité monoamine oxydasique a été inhibée, pourrait être une illustration du phénomène accru de diffusion. Cependant, là aussi, des recherches complémentaires sont nécessaires.

\section{Conclusion.}

Des recherches complémentaires effectuées dans l'organe pinéal de Brochet en histochimie de fluorescence, ultracytochimie (Falcon ef al., 1980) et radioautographie, il ressort que :

- le «pool » de 5-HTP/5-HT, ou d'une façon plus générale le « pool » d'indolamines${ }^{3} \mathrm{H}$, se répartił dans les différentes régions de l'organe pinéal, c'est-à-dire dans les zones où nous avions préalablement établi une différenciation sensorielle, mais aussi dans celle où la rudimentation qualitative des CTP ef quantitative des neurones s'est mise en place (Falcon, 1979b, c ; Falcon et Mocquard, 1979) ;

- les indolamines sont présentes dans les CTP et les CIE. Si une partie des CTP (photorécepteurs typiques) semble seulement être impliquée dans l'élaboration des messages sensoriels (Falcon et al., 1979b), en revanche toutes les CTP participent au stockage de la 5-HT et probablement à son métabolisme ;

- la dégradation, par désamination oxydative, de la 5-HT a lieu aussi bien dans les CTP que dans les CIE ;

- lorsque la désamination oxydative n'est pas, ou incomplèłement, inhibée expérimentalement, le taux de 5-HTP/5-HT (Falcon ef al., 1980) ou d'indolamines tritiées est régulièrement plus élevé dans les CTP que dans les CIE. La 5-HT na constitue pas un «pool » granulaire ou vésiculaire et ne semble pas être le neurotransmetteur des photorécepleurs typiques ;

- la participation la plus importante dans le métabolisme indolique revient aux CTP, propriété déjà reconnue dans les cellules homologues des Amniotes (Collin, 1979). Dans les CTP, à côté de la synthèse de la 5-HT, celle de la NAT et de l'HIOMT (conduisant à la formation de la mélatonine) est très plausible, mais ce n'est là qu'une hypothèse de travail ;

- l'existence de la 5-HT dans les CIE ne prouve pas que cette indolamine soit synthétisée dans ce type cellulaire. La rareté en structures de type vésiculaire (vésicules à cœur dense : Falcon, 1979b) est vraisemblablement très insuffisante pour assurer, comme chez certains Sauropsidés (Collin, 1979), la rétention de la 5-HT dans les CTP. Cette situation pourrait ainsi favoriser la diffusion de la 5-HT des CTP vers les CIE (voir discussion chez les Amniotes in Collin, 1979) ;

- compte tenu de la pluralité des CTP (Falcon, 1979b), le problème se pose de savoir si certaines propriétés biochimiques et en conséquence physiologiques, leur sont communes ou différentes. En d'autres termes, la voie de synthèse de la mélatonine, 
à partir de la $5-\mathrm{HT}$, existe-t-elle dans toutes les CTP ou seulement dans l'une des 2 catégories? La même question se pose pour le 5-méthoxytryptophol, un autre dérivé actif méthylé dont on ignore encore l'existence chez les Poissons.

De toute façon, cetie étude de l'organe pinéal du Brochet ne laisse pas apparaître qu'un message neuroendocrinien (de type indolique : mélatonine) remplace le message photosensoriel, présumé disparu dans certaines CTP (Falcon, 1979b). En effet, malgré les incertitudes mentionnées ci-dessus, il n'y a actuellement aucune incompatibilité à suggérer que certaines CTP (exemple : dans la région proximale) élaborent un double message, neurosensoriel et neurohormonal, alors que d'autres (exemple : dans la région médiane) auraient seulement perdu leur aptitude à transmettre le message sensoriel. Pour chaque type de CTP, se pose le problème de la régulation du métabolisme indolique.

Nos résultats sont complémentaires de ceux acquis antérieurement chez la Grenouille (réf. in Oksche, 1971 ; Oksche ef Hartwig, 1975, 1979 ; Dodt, 1973 ; Hamasaki et Eder, 1977). Chez cette espèce, dans les «sensory cells, an input of photic information might be converted in both electrical and neurohumoral outputs 》 (Oksche et Hartwig, 1975).

Ainsi, au cours de nos recherches ultérieures, nous viserons à développer la notion qu'une cellule de type pholorécepteur, à côté de messages neurosensoriels, est capable d'élaborer un (ou des) message(s) de type neurohormonal (comme la mélatonine) et tenterons ainsi d'établir le concept de cellule photoneuroendocrine (photo- : directement photosensible ; neuro- : dérivé du neuroectoderme ; endocrine : élaboration de neurohormones) proposé par Collin (1971), à partir du concept plus général des systèmes photoneuroendocriniens de Scharrer (1964).

Une combinaison de techniques appropriées (en préparation) devrait permettre d'aboutir à une importante notion de neurobiologie cellulaire, dans le domaine de la photoneuroendocrinologie. C'est seulement dans une étape suivante que l'étude du couplage stimulus-sécrétion (Collin, 1979 ; Collin et Oksche, en préparation) des cellules récepto-sécrétrices pinéaliennes offrira, de toute évidence, de nouvelles perspectives de recherche dans le domaine des mécanismes de régulation neuroendocrinienne (voir introduction).

Reçu en janvier 1980. Accepté en janvier 1980.

En fémoignage de vive gratitude, ce travail est dédié à Monsieur le Professeur J. AriënsKappers, à l'occasion de son $70^{\mathrm{e}}$ anniversaire.

Remerciements. - Nous remercions vivement les Professeurs Billard (Jouy-en-Josas), Follenius (Strasbourg), Fontaine (Paris) et Olivereau (Paris) qui, pour l'étude in vitro, nous ont guidé dans le choix des solutions physiologiques, et Mlle D. Decourt qui a assuré la dactylographie de ce mémoire. L'aide de J. Y. Driot, au cours de l'expérience effectuée en 1975, a été également très appréciée.

Ce travail a pu être réalisé grâce à une allocation d'éłude DGRST (attribuée à J. Falcon de 1976 à 1978) et à l'aide financière de la DGRST (contrat no 76.7.0043), du CNRS et de l'Université de Poitiers. 


\section{Références}

BALEMANS M. G. M., 1974. The pineal gland. A study on the presence and the biological significance of melatonin and 5-methoxytryptophol. Thesis, Utrecht.

BALEMANS M. G. M., 1979. Indole metabolism in the pineal gland of the rat ; some regulatory aspects Progr. Broin Res., 52, 221-228.

BEAUDET A., DESCARRIES L., 1978. The monoamine innervation of rat cerebral cortex : synaptic and nonsynaptic axon terminals. Neuroscience, 3, 851-860.

BOSLER O., 1978. Radioautographic identification of serotonin axon terminals in the rat organum vasculosum laminae terminalis. Brain Res., 150, 177-181.

BOUCHAUD C., ARLUISON M., 1977. Serotoninergic innervation of ependymal cells in the rat subcommissural organ. A fluorescence electron microscopic and radioautographic study. Biol. cell., 30, 65-72.

CALAS A., ALONSO G., ARNAULD E., VINCENT J. D., 1974. Demonstration of indolaminergic fibres in the median eminence of the duck, rat and monkey. Noture, Lond., 250, 242-243.

COLLIN J. P., 1969. Contribution à l'étude de l'organe pinéal. De l'épiphyse sensorielle à la glande pinéale : modalités de transformation et implications fonctionnelles. Ann. Stat. Biol. Besse-enChandesse, suppl. 1, 1-359.

COLLIN J. P., 1971. Differentiation and regression of the cells of the sensory line in the epiphysis cerebri, 79-120. In WOLSTENHOLME G. E. W., KNIGHT J., The pineal gland, Churchill Livingstone, Edinburgh-London.

COLLIN J. P., 1977. La rudimentation des photorécepteurs dans l'organe pinéal des Vertébrés, 393-407. In RAYNAUD A., Mécanismes de la rudimentation des organes chez les embryons de Vertébrés, Coll. int. C.N.R.S., nº 266, Paris.

COLLIN J. P., 1979. Recent advances in pineal cytochemistry. Evidence of the production of indoleamines and proteinaceous substances by rudimentary photoreceptor cells and pinealocytes of Amniota. Progr. Brain Res., 52, 271-296.

COLLIN J. P., MEINIEL A., 1973a. Métabolisme des indolamines dans l'organe pinéal de Lacerta (Reptiles, Lacertiliens). I. Intégration sélective de $5-\mathrm{HTP}-{ }^{3} \mathrm{H}$ (5-hydroxytryptophane- ${ }^{3} \mathrm{H}$ ) et rétention de ses dérivés dans les photorécepteurs rudimentaires sécrétoires. $Z$. Zellforsch., 142, 549-570.

COLLIN J. P., MEINIEL A., 1973b. Métabolisme des indolamines dans l'organe pinéal de Lacerfa (Reptiles, Lacertiliens). Il. L'activité MAO et l'incorporation de $5-\mathrm{HTP}-{ }^{3} \mathrm{H}$ et de $5-\mathrm{HT}-{ }^{3} \mathrm{H}$, dans les conditions normales et expérimentales. $Z$. Zellforsch., 145, 331-361.

COLLIN J. P., OKSCHE A. Structural and functional relationships in the nonmammalian pineal organ (en préparation).

COLLIN J. P., CALAS A., JUILLARD M. T., 1976. The avian pineal organ. Distribution of exogenous indoleamines: a qualitative study of the rudimentary photoreceptor cells by electron microscopic radioautography. Exp. Brain Res., 25, 15-33.

DE VLAMING V., VODICNIK M. J., 1978. Seasonal effects of pinealectomy on gonadal activity in the goldfish, Corassius auratus. Biol. Reprod., 19, 57-63.

DODT E., 1973. The parietal eye (pineal and parietal organs) of lower vertebrates, 113-140. In JUNG R., Handbook of sensory physiology, Vol. VII/3B, Central visual information B, Springer Verlag, Berlin, New York.

FALCON J., 1979a. L'organe pinéal du Brochet (Esox lucius, L.). I. Etude anatomique ef cytologique. Ann. Biol. anim. Bioch. Biophys., 19, 445-465.

FALCON J., 1979b. L'organe pinéal du Brochet (Esox lucius, L.). II. Etude en microscopie électronique de la différenciation et de la rudimentation partielle des photorécepteurs ; conséquences possibles sur l'élaboration des messages photosensoriels. Ann. Biol. anim. Bioch. Biophys., 19, 661-688.

FALCON J., 1979c. Unusual distribution of neurons in the pike pineal organ. Progr. Brain Res., 52, 89-91.

FALCON J., MOCQUARD J. P., 1979. L'organe pinéal du Brochet (Esox lucius, L.). III. Voies intrapinéales de conduction des messages pholosensoriels. Ann. Biol. anim. Bioch. Biophys., 19, 10431061. 
FALCON J., JUILLARD M. T., COLLIN J. P., 1980. L'organe pinéal du Brochet (Esox lucius, L.). IV. Sérotonine endogène et activité monoamine oxydasique ; étude histochimique, ultracytochimique ef pharmacologique. Reprod. Nutr. Dévelop., 20, 139-154.

FENWICK J. C., 1970. Demonstration and effect of melatonin in fish. Gen comp. Endocr., 14, 86-97.

GERN W. A., OWENS D. W., RALPH C. L., 1978a. The synthesis of melatonin by the trout retina. J. exp. Zool., 206, 263-270.

GERN W. A., OWENS D. W., RALPH C. L., 1978b. Persistence of the nycthemeral rhythm of melatonin secretion in pinealectomized or optic tract-sectioned trout (Salmo gairdneri). J. exp. Zool., 205, 371-376.

GERN W. A., RALPH C. L., 1979. Melatonin synthesis by the retina. Science, 204, 183-184.

GERSHON M. D., ROSS L. L., 1966. Radioisotopic studies of the binding, exchange, and distribution of 5-hydroxytryptamine synthesized from its radioactive precursor. J. Physiol. (Lond.), 186, 451-476.

HAFEEZ M. A., ZERIHUN L., 1976. Autoradiographic localization of ${ }^{3} \mathrm{H}-5-\mathrm{HTP}$ and ${ }^{3} \mathrm{H}-5-\mathrm{HT}$ in the pineal organ and circumventricular areas in the rainbow trout, Salmo gairdneri, Richardson. Cell Tiss. Res., 170, 61-76.

HAMASAKI D. I., EDER D. J., 1977. Adaptative radiation of the pineal system, 497-548. In CRESCITELLI F., Handbook of sensory physiology, Vol. VII/5 ; The visual system in vertebrates, SpringerVerlag, Berlin, Heidelberg, New York.

JUILLARD M. T., COLLIN J. P., 1979. Membranous sites of oxidative deamination : a comparison between ultracytochemical and radioautographic studies in the pineal organ of the wall lizard and the parakeet. Biol. cell., 36, 29-35.

LARRA F., DROZ B., 1970. Techniques radioautographiques et leur application à l'étude du renouvellement des constituants cellulaires. J. Microsc., 9, 845-880.

LERNER A. B., CASE J. D., TAKAHASHI Y., LEE T. H., MORI W., 1958. Isolation of melatonin, the pineal gland factor that lightens melanocytes. J. amer. Chem. Soc., 80, 2587.

OKSCHE A., 1971. Sensory and glandular elements of the pineal organ, 127-146. In WOLSTENHOLME G. E. W., KNIGHT J., The pineal gland, Churchill Livingstone, Edinburg-London.

OKSCHE A., HARTWIG H. G., 1975. Photoneuroendocrine systems and the third ventricle, 40-53. In KNIGGE K. M., SCOTT D. E., KOBAYASHI H., ISHII S., Brain endocrine inferaction. II. The Ventricular system. S. Karger A. G., Basel.

OKSCHE A., HARTWIG H. G., 1979. Pineal sense organs : components of photoneuroendocrine systems. Prog. Brain Res., 52, 113-130.

QUAY W. B., 1965. Retinal and pineal hydroxyindole-0-methyl transferase activity in vertebrates. Life Sci., 4, 983-991.

QUAY W. B., 1974. Pineal chemistry. C. C. Thomas, Springfield, $430 \mathrm{pp}$.

RALPH C. L., 1978. Pineal control of reproduction : nonmammalian vertebrates. Prog. reprod. Biol., 4, 30-35.

SCHARRER E., 1964. Photoneuroendocrine systems : general concepts. Ann. N. Y. Acad. Sci., 117, 13-22.

SMITH J. R., WEBER L. J., 1973. Acetylserotonin methyltransferase (ASMT) activity in the pineat gland and retina of rainbow trout. Proc. West. Pharmacol. Soc., 16, 191-194.

SMITH J. R., WEBER L. J., 1974. Diurnal fluctuations in acetylserotonin methyltransferase (ASMT) activity in the pineal gland of the steelhead trout (Salmo gairdneri). Proc. Soc. exp. Biol. Med., 147, $441-443$.

SMITH J. R., WEBER L. J., 1976. The regulation of day-night changes in hydroxyindole-0-methyltransferase activity in the pineal gland of steelhead trout (Salmo gairdneri). Can. J. Zool., 54, 1530-1534.

VODICNIK M. J., DE VLAMING V. L., 1978. The effects of pinealectomy on pituitary prolactin levels in Carassius auratus exposed to various photoperiod-femperature regimes. Endocr. Res. Com., $5,199-210$.

WILHOFT D. C., QUAY W. B., 1965. Effects of temperature on brain contents of 5-hydroxytryptamine and related indoles in a lizard, Sceloporus occidentalis. Comp. Biochem. Physiol., 15, $235-$ 338.

WURTMAN R. J., AXELROD J., KELLY D. E., 1968. The pineal. Acad. Press, New York, 199 pp. 